\title{
Dengue fever on the rise at tourist getaways
}

Published at www.cmaj.ca on Jan. 21

$\mathrm{T}$ he destinations read like a sunlover's dream: Acapulco, Puerto Vallarta, the Caribbean and Australia, among other winter tourist getaways.

But, in fact, they are destinations that the Public Health Agency of Canada (PHAC) warns put travellers at higher risk of contracting dengue fever, the incidence of which is rising as a consequence of such factors as increased international travel and, some say, global warming.

"This is a nasty disease" and the increase in cases is a major concern, says microbiologist Dr. Brian Ward. "Dengue is likely the worst illness you're likely to get from many tourist destinations."

Ward, the associate director of the J.D. MacLean Centre for Tropical Diseases at McGill University in Montréal, Quebec, speaks from personal experience. He contracted dengue fever while working as a volunteer in a refugee camp in Thailand in 1985.

"It hurts like hell," Ward says. "The Thai common name for the disease is 'breakbone fever' and you feel like your bones are being crushed. So most people are prostrate with it. You feel really crappy and basically don't want to do anything. Most people will have quite a severe headache."

Dengue is a febrile disease transmitted to humans by infected mosquitos, particularly Aedes aegpyti, which breeds in standing water often found in urban areas. The virus typically has a 5-10 day incubation period, after which there can be abrupt onset of headache, muscle and joint pain, fever and rash.

"And you can get it four times because there are four serotypes of dengue," Ward says. "The first time you get it, you're unlikely to get any of the very serious complications. But if the timing is just right, the second time you get dengue ... you can actually

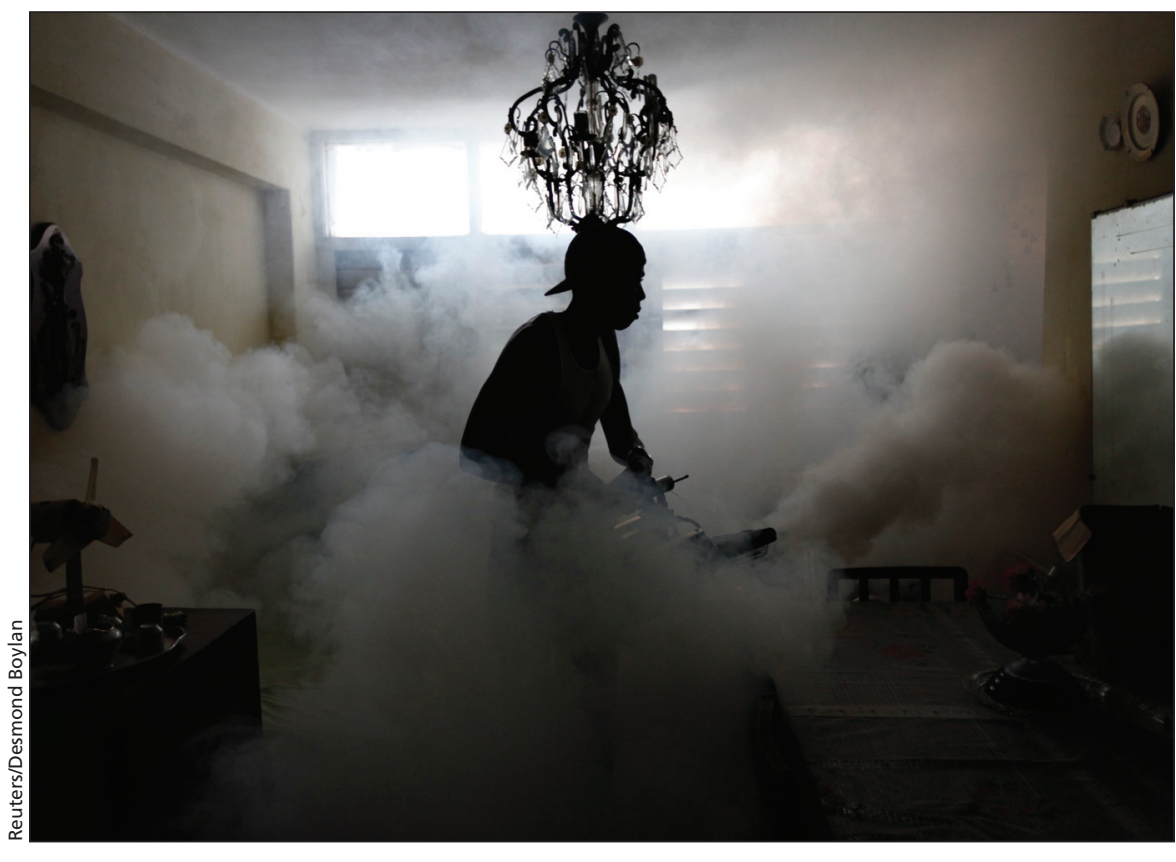

A member of a fumigation brigade sprays a home in Havana, Cuba. The government of Cuba conducts regular fumigation inside homes to contain the spread of dengue fever.

develop dengue hemorrhagic fever which can be lethal."

Symptoms for dengue hemorrhagic fever include loss of appetite, vomiting, intense abdominal pain, shock and bleeding from the nose or under the skin. A small number of cases lead to dengue shock syndrome, for which there is a high mortality rate.

Treatment is typically supportive therapy, with increased oral fluid intake recommended to prevent dehydration.

While dengue fever is traditionally thought of as a tropical disease and is endemic in more than 100 countries, experts say there have recently been huge outbreaks in Puerto Rico, northern Australia (its worst outbreak in 50 years) and Mexico. In 2009, it appeared for the first time in 40 years in Florida and for the first time ever in Cape Verde, off the western coast of Africa.

"Certainly travel is a significant factor," says Dr. Danielle Grondin, director-general of PHAC's infectious disease and emergency preparedness branch. "The sheer volume of travellers, the speed of travel. A person may get infected during travel, but the incubation period is longer than the travel time ... so they may get the symptoms back in their own country."

PHAC estimates that in the past 25 years, outbreaks of the virus have increased to the point where about $40 \%$ of the world's population now lives in areas where there is a risk of getting it.

Ward says global warming "may be contributing to its spread into areas like southern Florida and Australia. But mosquitoes also need places to breed. And these mosquitoes are very talented at breeding in urban settings. So a tin can. A garden shed bucket. ... Also, in the last little while, in many places in the world we've said it's illegal to use [pesticide] DDT. Many people attribute the resurgence of malaria in Africa to the greater difficulties in getting and using DDT. DDT was effective not just with malaria-bearing mosquitoes but also with dengue-bearing mosquitoes." 
Ward also forecasts further encroachments in the United States because of its proximity to Puerto Rico. "Diseases spread and the more movement you have between places the more likely it is to spread. The continental US and Puerto Rico are pretty darned close so a huge outbreak in Puerto Rico is very, very likely to have things move up into the southern US."

Grondin says tourists should prepare themselves by packing insect repellent, proper clothing and sleeping nets, particularly as there is no vaccine for dengue fever. "If you're just going to a resort in Acapulco and you're never going off the resort, you're probably fine. But unless the hotel has very large grounds and does a lot of insect control on its grounds, mosquitoes don't care if you're standing in Hedonism II or in a shack. You're just food." - Becky Rynor, Ottawa, Ont.

DOI:10.1503/cmaj.109-3169 